\title{
Seeing is Believing - Enhancing Message Dissemination in Vehicular Networks Through Visual Cues
}

\author{
Mate Boban ${ }^{1,2}$ Tiago T. V. Vinhoza ${ }^{2}$, Ozan K. Tonguz ${ }^{1}$ and João Barros ${ }^{2}$ \\ \{mboban@cmu.edu, tiago.vinhoza@ieee.org, tonguz@ece.cmu.edu, jbarros@fe.up.pt\}
}

\begin{abstract}
One of the stumbling blocks for implementation of Vehicular Ad Hoc Networks is the penetration rate: the percentage of vehicles that have the communication equipment installed. As the equipment deployment is unlikely to happen instantaneously, it is important to explore the performance gains achievable at low penetration rates. This especially pertains to safety applications, which are expected to provide life-saving information to all drivers on the road within a given region. We propose a technique that can be employed by safety applications to address the low penetration issue. By using visual cues on the equipped vehicles, such as specific patterns of hazard warning lights, we show that for all but the lowest vehicle densities, a radio penetration rate of $30 \%$ is sufficient to inform more than $95 \%$ of drivers in the region of interest in a timely manner.
\end{abstract}

Index Terms-Vehicular ad hoc networks, vehicle-to-vehicle communication, safety applications, visual cues.

\section{INTRODUCTION}

Vehicular Ad Hoc NETworks (VANETs) are designed with the primary goal of decreasing the number of road accidents. By using radio communication equipment (e.g., compliant with the IEEE 802.11p [1] standard), vehicles will have the ability to exchange life-saving information about hazardous situations in a timely manner, thereby assisting the drivers in avoiding accidents.

Deployment of VANET communication equipment in vehicles will be gradual, with an estimated time of approximately 15 years before all vehicles are equipped [2]. Therefore, during the deployment phase, the penetration rate will be below $100 \%$, which is often deemed as necessary for successful implementation of safety applications, such as those discussed in [3]. To help overcome this issue, we propose to combine standard wireless communication with a visual cue technique that helps inform the drivers of the unequipped vehicles about hazardous situations.

The first collaborative safety application on highways resulted from manually switching on the hazard warning lights to inform other drivers about a dangerous situation ahead, such as slow or halted vehicle, an accident, or adverse weather conditions. Inspired by this visual cue technique, we propose to leverage radio communication to exchange information about the hazardous situation among the radio-equipped vehicles, at the same time automatically activating visual cues

\footnotetext{
${ }^{1}$ Department of Electrical and Computer Engineering, Carnegie Mellon University, USA

${ }^{2}$ Instituto de Telecomunicações, Faculdade de Engenharia da Universidade do Porto, Portugal

This work was funded in part by the Portuguese Foundation for Science and Technology under the Carnegie Mellon | Portugal program (grant SFRH/BD/33771/2009) and the DRIVE-IN project (CMUPT/NGN/0052/2008. http://drive-in.cmuportugal.org).
}

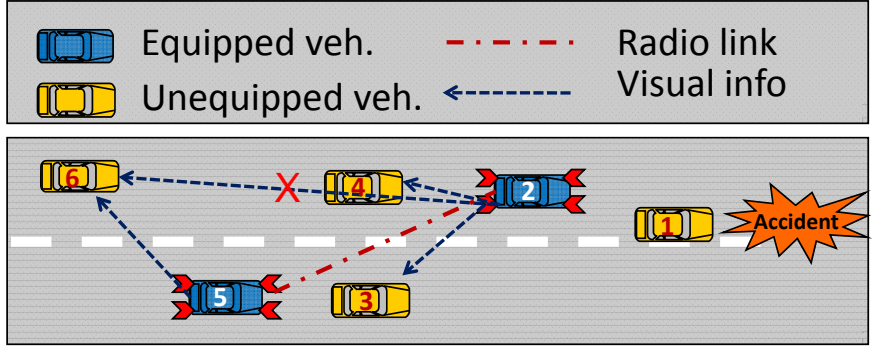

Fig. 1. Example of the proposed technique. All vehicles are traveling left to right. Vehicles $1,3,4$, and 6 are not equipped with IEEE $802.11 \mathrm{p}$ radios, whereas vehicles 2 and 5 are equipped. Vehicle 1 is caught in an accident. Assume that only vehicle 2 is in the visual range of the accident when it happens. Vehicle 2 detects the hazardous situation through the driver's reaction (e.g., strong braking). Vehicle 2 turns on the warning lights automatically and immediately informs the driver in vehicle 5 through the radio link. Vehicle 5 turns on the warning lights automatically. Drivers in vehicles 3 and 4 get visual cues from vehicle 2. Driver in vehicle 6 cannot see vehicle 2, but gets a visual cue from vehicle 5 .

on the equipped vehicles to inform the drivers of unequipped vehicles. This way, the visual cues are serving as a substitute for the in-vehicle notifications that are available to the drivers in the equipped vehicles, as shown in Fig. 1] The visual cues can be simple modifications of frequency or intensity of the hazard warning lights or the stop lights 1 Depending on the safety application at hand (e.g., accident ahead, oil or ice on the road), the automatic warning system decides on the type of visual cue and the size of the information dissemination area. The proposed visual cue technique could be easily adopted by a number of safety applications, such as those identified by European Telecommunications Standards Institute [4]: collision risk warning, stationary vehicle, wrong way driving warning, signal violation warning, among others. More specifically, the technique is useful for applications related to hazard notification and collision avoidance [5], where it can help visually inform more drivers than the radio communication alone.

A number of studies have been conducted to evaluate the benefits of hazard notification and collision avoidance systems. Some studies focused on the performance of hazard notification systems in low penetration rate scenarios (e.g., [6]), while others focused on experimental evaluation of collision avoidance systems (e.g., [7]). To the best of our knowledge, none of the existing studies proposed utilizing visual cues on the equipped vehicles to complement the radio communication

\footnotetext{
${ }^{1}$ Similar visually distinguishable cues are already making their way in personal vehicles, in the form of emergency stop signals, which adjust the frequency and intensity of hazard or stop lights in case of severe deceleration.
} 
in informing a larger number of drivers about a hazardous situation.

\section{SySTEM MODEL}

The proposed warning scheme is depicted in Fig. 1. We assume that, if the radio-equipped vehicles are not already aware of the danger, once within the visibility range of the hazardous situation they will automatically detect it, either through the behavior of the vehicle (e.g., skidding, loss of traction) or through the reaction of the driver (e.g., sudden braking). This is in line with the behavior of the proposed VANET safety applications [4]. Once informed, the equipped vehicles will automatically start notifying the unequipped vehicles through visual cues without the aid of the driver. At the same time, using radio communication, equipped vehicles are able to inform other equipped vehicles, which automatically turn on their warning lights thereby giving visual cues to the unequipped vehicles. The proposed warning scheme does not assume any cooperation from human drivers in unequipped vehicles (e.g., by manually turning their warning lights on).

\section{A. Environment Variables}

We simulate the effectiveness of the proposed technique in different visibility conditions. We use two thresholds: a 40 meters visibility range designated in Boussard et al. [8] as low visibility (e.g., under foggy conditions), and 100 meters, designated by governmental bodies as the minimum required visibility range for hazard warning lights in normal sunlight (e.g., see [9]). In order for the driver to see a visual cue within these ranges, the following prerequisites need to be met:

- There needs to exist a vehicle in front with its visual cues turned on;

- Visual line-of-sight (LOS) needs to exist (i.e., there are no other vehicles without the visual cues that are obstructing the LOS between the vehicle in front with the visual cues and the vehicle that needs to be informed).

Only if the above prerequisites are met, the driver can see the visual cues.

On highways, we assume that only vehicles driving in the same direction are visible (road separators might occlude visibility for the drivers traveling in the opposite direction). On rural roads, we assume that the vehicles traveling in the opposite direction are visible (i.e., the road has no separators).

We define the danger zone as the area within a 250 meter radius of the hazardous location, which resembles the breaking distance for a vehicle traveling at $100 \mathrm{~km} / \mathrm{h}$ in highly adverse (wet and icy) conditions [10]. We tested different danger zone radii, ranging from 50 meters to 300 meters; we did not observe significant performance differences.

Furthermore, we simulate two distinct hazardous scenarios: lead-vehicle stationary (LVS) and lead-vehicle moving (LVM). In LVS, there is a stopped vehicle on the road (resembling an accident or a stalled vehicle). When the lead vehicle is moving (LVM), there is a point on the road where a hazardous situation occurs, but there is no stopped vehicle (such situations occur in case of an oil spill, icy road, or a similar road hazard). These two scenarios result in significantly different vehicular mobility dynamics (e.g., closing speeds and distances).
TABLE I

Simulation AND ENVIRONMENT PARAMETERS

\begin{tabular}{|ll|}
\hline Parameter & Values \\
\hline \hline Road types & $\begin{array}{l}\text { Rural (1 lane per direction) } \\
\text { Highway (2, 3 lanes per direction) }\end{array}$ \\
\hline Road length & $13.5 \mathrm{~km}$ \\
\hline Vehicle density (veh/km/lane) & Low (2.5), Med. (7.5), High (12.5) [13] \\
\hline Vehicle ratios & Personal: 85\% Commercial: 15\% [1] \\
\hline Simulation duration & 150 seconds per run \\
\hline Number of simulation runs & 100 per scenario \\
\hline \hline Visibility ranges & Low: 40 m [8], Normal: 100 m [9] \\
\hline Driver reaction time & Uniform between 1 and 3 seconds $[14]$ \\
\hline Hazardous scenarios & LVM - Lead Vehicle Moving \\
& LVS - Lead Vehicle Stationary [15] \\
\hline \hline IEEE 802.11p radio settings & Tx Pwr: 20 dBm, Rx Sens: -90 dBm \\
\hline
\end{tabular}

\section{B. Simulation Parameters}

Since the exact vehicle dimensions and locations are important for accurate evaluation of the proposed technique, the vehicle dimensions (height, width, length) and the ratio of personal vehicles (passenger cars) and commercial vehicles (vans, trucks, buses, etc.) are based on the empirical data reported in [11]. Using the empirical data, we generate vehicular mobility traces with the STRAW (STreet RAndom Waypoint) mobility model [12]. STRAW implements the car-following model with lane changing, intersection control, and lane offset (the variation in location of the vehicle with respect to the center of the lane). To verify the realism of the generated vehicular traces, we compared the traces with the aerial photography of A28 highway near Porto, Portugal (dataset described in detail in [11]). We observed a very good agreement between the cumulative distribution functions of the inter-vehicle spacing for the generated traces and for the A28 highway.

We employ the radio propagation model described in [11]. The model accounts for vehicles as three-dimensional obstacles and takes into account their impact on the received signal power and the packet reception rate. We also simulate frame collisions at the medium access layer, in order to check whether the medium contention and the induced frame loss have a significant impact on the efficacy of the proposed visual cue technique. To resolve the collisions, we assume that the application reschedules the transmissions if an acknowledgement is not received within 200 milliseconds.

The remaining environment and simulation parameters are presented in Table \

\section{RESUlTS AND DISCUSSION}

Figure 2 shows the ratio of drivers informed before reaching the danger zone on a two lane highway for low, medium, and high vehicle density, in normal visibility for LVM (Fig. 2a) and LVS (Fig. 2b) scenarios. For both LVS and LVM, in normal visibility and at $20 \%$ radio penetration rate, $90 \%$ of the drivers were informed in low vehicle density scenarios. In medium and high vehicle density scenarios, above $95 \%$ of drivers were informed. At $30 \%$ penetration rate, across all densities more than $95 \%$ of the drivers were informed about the hazardous situation on time 2 To inform the same percentage of drivers

\footnotetext{
${ }^{2}$ Since LVM and LVS scenarios exhibited similar results, from this point onward we mainly focus on LVM scenarios.
} 


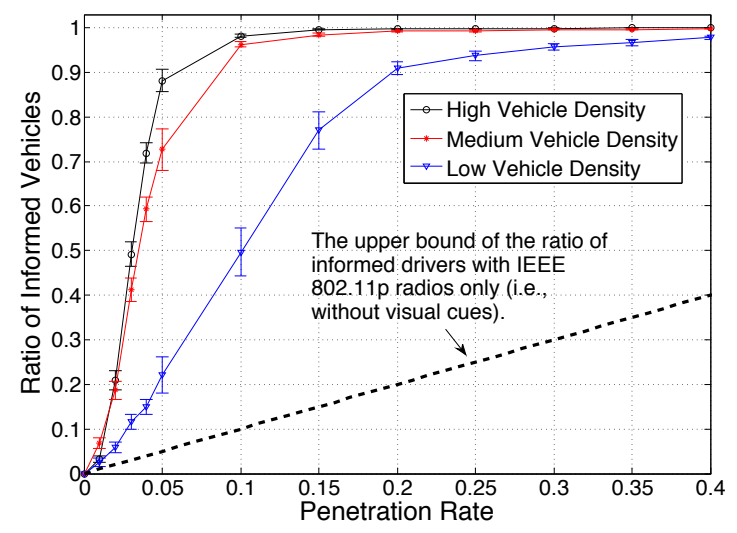

(a) LVM, normal visibility.

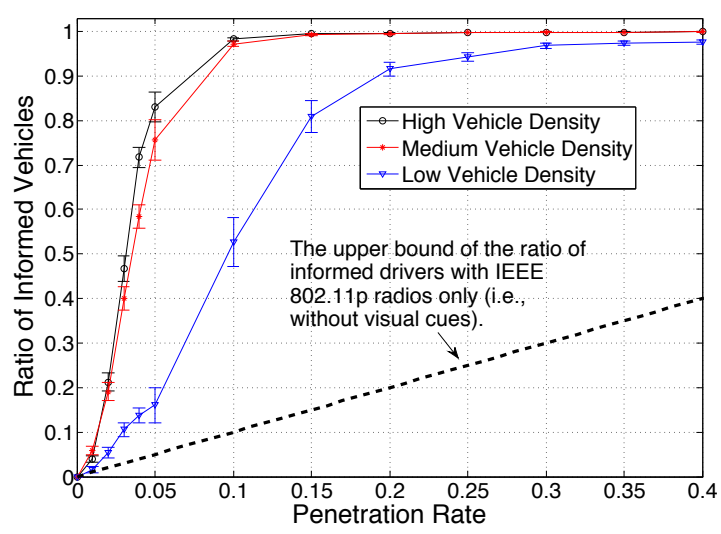

(b) LVS, normal visibility.

Fig. 2. The ratio of drivers (in both equipped and unequipped cars) informed about the hazardous situation before they enter the danger zone in case of a highway with two lanes per direction. The error bars represent the 95\% confidence bounds. The three vehicle densities (high, medium, and low) are based on the values reported in [13].

in the reduced visibility conditions, the required penetration rate becomes $40 \%, 30 \%$ and $25 \%$ for low, medium, and high densities, respectively (Table [II). Higher vehicle densities are beneficial because the larger absolute number of equipped vehicles increases the likelihood that the drivers of unequipped vehicles will be exposed to visual cues. For low density and low penetration rate, the number of equipped vehicles is simply too low. This results in a large inter-vehicle distance between the equipped vehicles, which effectively leads to a disconnected network.

Figure 2 refers only to a two lane per direction highway. We also analyzed three lane highways as well as one lane rural roads. The results for the selected scenarios are presented in Table [I] The ratio of informed drivers is directly dependent on the density of vehicles in an area (i.e., across all lanes), with virtually all drivers informed on a three lane highway at $10 \%$ penetration rate for medium and high vehicle density scenarios, and at $20 \%$ for low vehicle density scenarios. Due to the smaller absolute number of vehicles on a rural road, penetration rates of $15 \%, 20 \%$, and $40 \%$ are required for low, medium, and high density, respectively.

The amount of time that drivers have between the time they are informed about the hazardous situation and when they reach the danger zone is shown in Fig. 3. The figure depicts one specific scenario; however, other vehicle densities and penetration rates exhibit similar behavior. As can be seen from the figure, virtually all vehicles that are outside the danger zone at the time a hazardous situation occurs get notified on time. The large spread in time for a given distance implies that some drivers are informed through radio communication, some through visual cues, and others through the combination of the two (i.e., a single or multi-hop radio communication with a visual cue at the end).

The above results did not account for losses due to medium access contention (i.e., there is no interference that causes contention and collisions). To evaluate the efficacy of the proposed visual cue technique in the presence of interference and frame loss, we introduced the frame loss rates of $50 \%$ and $75 \%$, and we tested these loss rates at both low and
TABLE II

RADIO PENETRATION RATES AT WHICH MORE THAN 90\% OF DRIVERS ARE INFORMED FOR DIFFERENT ROAD AND VISIBILITY LVM SCENARIOS.

\begin{tabular}{|cccc|}
\hline Road type & $\begin{array}{c}\text { Veh. } \\
\text { Density }\end{array}$ & $\begin{array}{c}\text { Radio } \\
\text { Pen. Rate }\end{array}$ & $\begin{array}{c}\text { Percentage of informed } \\
\text { (95\% Conf. Bounds) }\end{array}$ \\
\hline \hline One lane & Low & $40 \%$ & {$[90 \%-100 \%]$} \\
Normal visibility & Medium & $20 \%$ & {$[95 \%-100 \%]$} \\
& High & $15 \%$ & {$[98 \%-99 \%]$} \\
\hline \hline Three lane & Low & $20 \%$ & {$[97 \%-99 \%]$} \\
Normal visibility & Medium & $10 \%$ & {$[99 \%-100 \%]$} \\
& High & $10 \%$ & {$[99 \%-100 \%]$} \\
\hline \hline Two lane & Low & $40 \%$ & {$[96 \%-97 \%]$} \\
Low visibility & Medium & $30 \%$ & {$[92 \%-94 \%]$} \\
& High & $25 \%$ & {$[96 \%-98 \%]$} \\
\hline
\end{tabular}

higher radio penetration rates (up to 60\%). The results in Table [III show that the effect of the frame loss on the overall performance of the proposed visual cue technique is negligible. The difference in the percentage of the informed drivers with and without frame loss was less than $1 \%$ for any given penetration rate. We explain the robustness of the technique with respect to the packet loss as follows. For the majority of vehicles, the amount of time before they reach the danger zone is large (see Fig. 3). Therefore, although the frame loss might obstruct the communication and cause additional delay in the propagation of visual cues for a certain period of time, that period of time is almost always shorter than the time it takes for a vehicle to reach the danger zone. In other words, by the time most vehicles reach the danger zone, they had had enough time to receive the (retransmitted) messages. Consequently, the additional packet loss only affects the vehicles in or very close to the danger zone.

\section{CONClusions AND Future Work}

We proposed a new technique for efficiently delivering safety-related information using both the visual and the radio communication channel in VANETs. In medium and high vehicle density scenarios, $10 \%-20 \%$ radio penetration rate in normal visibility (25\%-30\% in low visibility) is sufficient to inform more than $90 \%$ of the drivers, irrespective of the type 


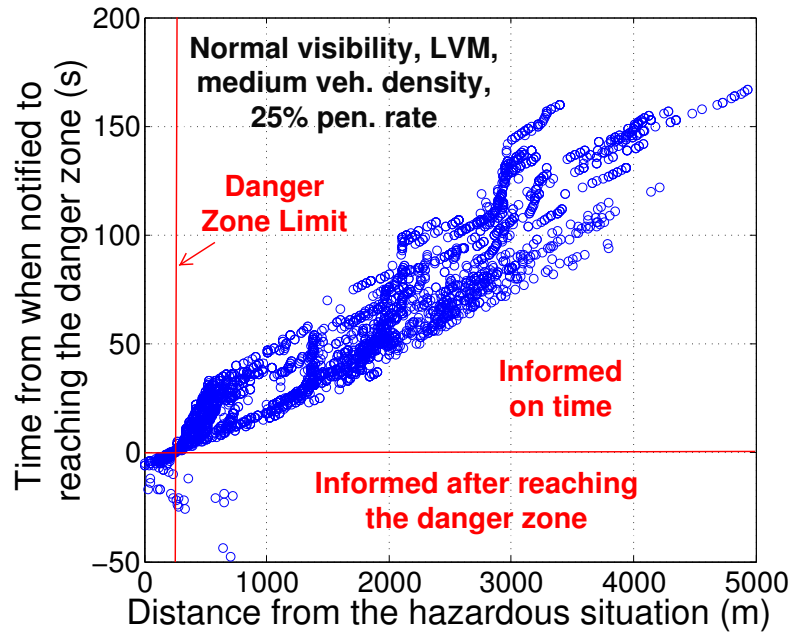

Fig. 3. The amount of time from the point when the driver is notified about the hazardous situation until he reaches the danger zone. The time is plotted as a function of the distance from the hazardous situation at the time when the hazardous situation happened. The negative time represents the drivers who were informed after reaching the danger zone. Each circle represents one vehicle.

TABLE III

PERFORMANCE OF THE PROPOSED VISUAL CUE TECHNIQUE IN THE PRESENCE OF INTERFERENCE AND FRAME COLLISIONS/LOSS FOR NORMAL VISIBILITY, MEDIUM VEHICLE DENSITY, TWO LANE, LVM SCENARIO.

\begin{tabular}{|ccc|}
\hline $\begin{array}{c}\text { Radio } \\
\text { Pen. Rate }\end{array}$ & $\begin{array}{c}\text { Percentage of informed } \\
\text { (95\% Conf. Bounds) } \\
\text { 50\% Frame Loss Rate }\end{array}$ & $\begin{array}{c}\text { Percentage of informed } \\
\text { (95\% Conf. Bounds) } \\
\mathbf{7 5 \%} \text { Frame Loss Rate }\end{array}$ \\
\hline $10 \%$ & {$[96.1 \%-97.4 \%]$} & {$[95.3 \%-96.8 \%]$} \\
$15 \%$ & {$[98.2 \%-99.0 \%]$} & {$[98.3 \%-99.0 \%]$} \\
$35 \%$ & {$[99.8 \%-99.9 \%]$} & {$[99.7 \%-99.9 \%]$} \\
$40 \%$ & {$[99.7 \%-100 \%]$} & {$[99.8 \%-99.9 \%]$} \\
$60 \%$ & {$[99.9 \%-100 \%]$} & {$[99.9 \%-100 \%]$} \\
\hline
\end{tabular}

of road. In low vehicle density scenarios the same is achieved with $20 \%-30 \%$ penetration ( $40 \%$ in low visibility). Also, we have shown that the proposed visual cue technique is robust in the presence of interference and frame collisions. The reported results suggest that, by employing the proposed technique, many of the proposed VANET safety applications could be supported in a cost-effective manner long before all the vehicles are equipped with radios. Furthermore, implementing such a technique could facilitate faster adoption of VANET technology, since life-saving functionalities could be achieved earlier.

The next steps needed for enabling the proposed technique in the real world are as follows.

- Characterization and classification of different safety applications that can be supported by the technique, based on the hazardousness of the situations; for example, minor hazard due to slowed traffic on the road ahead, medium hazard due to a stopped vehicle ahead and highly hazardous situation due to an accident immediately ahead.

- Based on the classification, rules for the information dissemination (both radio-based and visual) need to be set up, along with the duration, visual intensity, and the dissemination area (region of interest). This will help avoid the occurrence of both false-negatives (i.e., a hazardous situation was not reported or was deemed less dangerous than it was) and false-positives (i.e., a nonexistent or lowhazard situation was deemed dangerous).

- The analysis of human factors involved in reacting to the received information needs to be performed (e.g., drivers' reactions to the advisory/warning visual stimulus [15] and the optimal types of visual cues).

\section{REFERENCES}

[1] “IEEE Draft Standard IEEE P802.11p/D9.0," Tech. Rep., July 2009.

[2] F. Bai and B. Krishnamachari, "Exploiting the Wisdom of the Crowd: Localized, Distributed Information-Centric VANETs," IEEE Communications Magazine, vol. 48, no. 5, pp. 138 -146, May 2010.

[3] F. Bai, T. Elbatt, G. Hollan, H. Krishnan, and V. Sadekar, "Towards Characterizing and Classifying Communication-based Automotive Applications from a Wireless Networking Perspective," 1st IEEE Workshop on Automotive Networking and Applications (AutoNet), 2006.

[4] ETSI TC ITS, "Intelligent Transport Systems (ITS); Vehicular Communications; Basic Set of Applications; Definitions," Tech. Rep. ETSI TR 102638 V1.1.1, June 2009.

[5] S. Biswas, R. Tatchikou, and F. Dion, "Vehicle-to-vehicle Wireless Communication Protocols for Enhancing Highway Traffic Safety," IEEE Communications Magazine, vol. 44, no. 1, pp. 74 - 82, Jan. 2006.

[6] A. Lambert, D. Gruyer, A. Busson, and H. Mansoor Ali, "Usefulness of Collision Warning Inter-vehicular System," Int. J. Vehicle Safety, vol. 5, no. 1 , pp. $60-74,2010$.

[7] S. Demmel, D. Gruyer, J. Besnier, I. Ben Jemaa, S. Pechberti, and A. Rakotonirainy, "Collision Warning Dissemination in Vehicles Strings: An Empirical Measurement," in IEEE Intelligent Vehicles Symposium (IV), 2011, June 2011, pp. $454-459$.

[8] C. Boussard, N. Hautière and B. d'Andréa-Novel, "Visibility Distance Estimation Based on Structure From Motion," 11th International Conference on Control Automation Robotics Vision, pp. 1416 -1421, December 2010.

[9] State of Florida, "Statute 316.234, Signal Lamps and Signal Devices," 2010.

[10] L. Strandberg, "Winter Braking Tests with 66 Drivers, Different Tyres and Disconnectable ABS," in International Workshop on Traffic Accident Reconstruction, Tokyo, November 1998.

[11] M. Boban, T. T. V. Vinhoza, J. Barros, M. Ferreira, and O. K. Tonguz, "Impact of Vehicles as Obstacles in Vehicular Ad Hoc Networks," IEEE J. Select. Areas Commun., vol. 29, no. 1, pp. 15-28, January 2011.

[12] D. R. Choffnes and F. E. Bustamante, "An Integrated Mobility and Traffic Model for Vehicular Wireless Networks," VANET '05: Proceedings of the 2nd ACM international workshop on Vehicular ad hoc networks, pp. 69-78, 2005.

[13] V. Naumov, R. Baumann, and T. Gross, "An Evaluation of Inter-vehicle Ad Hoc Networks Based on Realistic Vehicular Traces," MobiHoc '06: Proceedings of the 7th ACM international symposium on Mobile ad hoc networking and computing, pp. 108-119, 2006.

[14] D. V. McGehee, E. N. Mazzae, and G. Baldwin, "Driver Reaction Time in Crash Avoidance Research: Validation of a Driving Simulator Study on a Test Track," IEA 2000/HFES 2000, vol. 44, no. 20, pp. 320-323, 2000.

[15] "IVHS Countermeasures for Rear-End Collisions, Task 1 Volume VI: Human Factors Studies," U.S. Department of Transportation, NHTSA, Tech. Rep. DOT HS 808 565, 1994. 